\title{
Numerical Analysis of a Distributed Optimal Control Problem Governed by an Elliptic Variational Inequality
}

\author{
Mariela Olguín ${ }^{1}$ and Domingo A. Tarzia ${ }^{2}$ \\ ${ }^{1}$ Departamento de Matemática, EFB-FCEIA, Universidad Nacional de Rosario, Avenida Pellegrini 250, S2000BPT Rosario, Argentina \\ ${ }^{2}$ Departamento de Matemática-CONICET, FCE, Universidad Austral, Paraguay 1950, S2000FZF Rosario, Argentina
}

Correspondence should be addressed to Domingo A. Tarzia; dtarzia@austral.edu.ar

Received 29 July 2015; Accepted 3 November 2015

Academic Editor: Kanishka Perera

Copyright (c) 2015 M. Olguín and D. A. Tarzia. This is an open access article distributed under the Creative Commons Attribution License, which permits unrestricted use, distribution, and reproduction in any medium, provided the original work is properly cited.

The objective of this work is to make the numerical analysis, through the finite element method with Lagrange's triangles of type 1 , of a continuous optimal control problem governed by an elliptic variational inequality where the control variable is the internal energy $g$. The existence and uniqueness of this continuous optimal control problem and its associated state system were proved previously. In this paper, we discretize the elliptic variational inequality which defines the state system and the corresponding cost functional, and we prove that there exist a discrete optimal control and its associated discrete state system for each positive $h$ (the parameter of the finite element method approximation). Finally, we show that the discrete optimal control and its associated state system converge to the continuous optimal control and its associated state system when the parameter $h$ goes to zero.

\section{Introduction}

We consider a bounded domain $\Omega \subset \mathbb{R}^{n}$ whose regular boundary $\partial \Omega=\Gamma_{1} \cup \Gamma_{2}$ consists of the union of two disjoint portions $\Gamma_{1}$ and $\Gamma_{2}$ with meas $\left(\Gamma_{1}\right)>0$. We consider the following free boundary problem $(S)$ :

$$
\begin{aligned}
u & \geq 0 ; \\
u(-\Delta u-g) & =0 ; \\
-\Delta u-g & \geq 0 \\
& \text { in } \Omega ; \\
u & =b \quad \text { on } \Gamma_{1} ; \\
-\frac{\partial u}{\partial n} & =q \quad \text { on } \Gamma_{2},
\end{aligned}
$$

where the function $g$ in (1) can be considered as the internal energy in $\Omega, b$ is the constant temperature on $\Gamma_{1}$, and $q$ is the heat flux on $\Gamma_{2}$. The variational formulation of the above problem is given as follows: find $u=u_{g} \in K$ such that $\forall v \in K$

$$
a(u, v-u) \geq(g, v-u)_{H}-\int_{\Gamma_{2}} q(v-u) d s,
$$

where

$$
\begin{aligned}
V & =H^{1}(\Omega), \\
K & =\left\{v \in V: v \geq 0 \text { in } \Omega, v=b \text { on } \Gamma_{1}\right\}, \\
V_{0} & =\left\{v \in V: v=0 \text { on } \Gamma_{1}\right\}, \\
H & =L^{2}(\Omega), \\
Q & =L^{2}\left(\Gamma_{2}\right), \\
(u, v)_{Q} & =\int_{\Gamma_{2}} u v d s \quad \forall u, v \in Q, \\
(u, v)_{H} & =\int_{\Omega} u v d x \quad \forall u, v \in H, \\
a(u, v) & =\int_{\Omega} \nabla u \cdot \nabla v d x \quad \forall u, v \in V .
\end{aligned}
$$

We note that $a$ is bilinear, continuous, and symmetric on $V$ and a coercive form on $V_{0}$ [1]; that is to say, there exists a constant $\lambda>0$ such that

$$
a(v, v) \geq \lambda\|v\|_{V}^{2} \quad \forall v \in V_{0} .
$$


In [2], the following continuous distributed optimal control problem associated with $(S)$ or the elliptic variational inequality (3) was considered as follows.

Problem $(P)$. Find the continuous distributed optimal control $g_{\text {op }} \in H$ such that [3-5]

$$
J\left(g_{\mathrm{op}}\right)=\min _{g \in H} J(g)
$$

where the quadratic cost functional $J: H \rightarrow \mathbb{R}_{0}^{+}$is defined by

$$
J(g)=\frac{1}{2}\left\|u_{g}\right\|_{H}^{2}+\frac{M}{2}\|g\|_{H}^{2}
$$

with $M>0$, a given constant, and $u_{g}$ is the corresponding solution of the elliptic variational inequality (3) associated with the control $g$.

Several continuous optimal control problems are governed by elliptic variational inequalities, for example, the process of biological waste-water treatment; reorientation of a satellite by propellers; and economics: the problem of consumer regulation of a monopoly and so forth. There exists an abundant literature for optimal control problems governed by elliptic variational equalities or inequalities [6-12], for numerical analysis of variational inequalities or optimal control problems [13-16].

The objective of this work is to make the numerical analysis of the optimal control problem $(P)$ which is governed by the elliptic variational inequality (3) by proving the convergence of a discrete solution to the continuous optimal control problems.

In Section 2, we establish the discrete elliptic variational inequality (10) which is the discrete formulation of the continuous elliptic variational inequality (3), and we obtain that these discrete problems have unique solutions for all positive $h$. Moreover, on the adequate functional spaces these solutions are convergent when $h \rightarrow 0^{+}$to the solutions of the continuous elliptic variational inequality (3).

In Section 3, we define the discrete optimal control problem (31) corresponding to continuous optimal control problem (6). We prove the existence of a discrete solution for the optimal control problem $\left(P_{h}\right)$ for each parameter $h$ and we obtain the convergence of this family with its corresponding discrete state system to the continuous optimal control with the corresponding continuous state system of the problem $(P)$.

\section{Discretization of the Problem $(S)$}

Let $\Omega \subset \mathbb{R}^{n}$ be a bounded polygonal domain; $b$ a positive constant; and $\tau_{h}$ a regular triangulation with Lagrange triangles of type 1 , constituted by affine-equivalent finite elements of class $C^{0}$ over $\Omega, h$ being the parameter of the finite element approximation which goes to zero $[17,18]$. We take $h$ equal to the longest side of the triangles $T \in \tau_{h}$ and we can approximate the sets $V$ and $K$ by

$$
\begin{aligned}
V_{h} & =\left\{v_{h} \in C^{0}(\bar{\Omega}): v_{h} \in \mathbb{P}_{1}(T) \text { on } T, \forall T \in \tau_{h}\right\}, \\
V_{h 0} & =\left\{v_{h} \in V_{h}: v_{h}=0 \text { on } \Gamma_{1}\right\}, \\
K_{h} & =\left\{v_{h} \in V_{h}: v_{h} \geq 0, \quad v_{h}=b \text { on } \Gamma_{1}\right\},
\end{aligned}
$$

where $\mathbb{P}_{1}(T)$ is the set of the polynomials of degree less than or equal to 1 in the triangle $T$. Let $\Pi_{h}: V \rightarrow V_{h}$ be the corresponding linear interpolation operator and $c_{0}>0$ a constant (independent of the parameter $h$ ) such that, $\forall v \in$ $H^{r}(\Omega), 1<r \leq 2[17]$ :

$$
\begin{aligned}
& \left\|v-\Pi_{h}(v)\right\|_{H} \leq c_{0} h^{r}\|v\|_{r}, \\
& \left\|v-\Pi_{h}(v)\right\|_{V} \leq c_{0} h^{r-1}\|v\|_{r} .
\end{aligned}
$$

The discrete variational inequality formulation $\left(S_{h}\right)$ of system $(S)$ is defined as follows: find $u_{h g} \in K_{h}$ such that $\forall v_{h} \in K_{h}$

$$
a\left(u_{h g}, v_{h}-u_{h g}\right) \geq\left(g, v_{h}-u_{h g}\right)_{H}-\left(q, v_{h}-u_{h g}\right)_{\mathrm{Q}} .
$$

Theorem 1. Let $g \in H, b>0$, and $q \in Q$; then there exists unique solution of the problem $\left(S_{h}\right)$ given by the elliptic variational inequality (10).

Proof. It follows from the application of Lax-Milgram Theorem [1].

Lemma 2. Let $g_{1}, g_{2} \in H$ and $u_{h g_{1}}, u_{h g_{2}} \in K_{h}$ be the solutions of $\left(S_{h}\right)$ for $g_{1}$ and $g_{2}$, respectively; then one has that

(a) there exists a constant $C$ independent of $h$ such that

$$
\left\|u_{h g}\right\|_{V} \leq C, \quad \forall h>0
$$

(b) $\forall h>0$

$$
\left\|u_{h g_{2}}-u_{h g_{1}}\right\|_{V} \leq \frac{1}{\lambda}\left\|g_{2}-g_{1}\right\|_{H}
$$

(c) if $g_{n} \rightarrow g$ in $H$ weak, then $u_{h g_{n}} \rightarrow u_{h g}$ in $V$ strong for each fixed $h>0$.

Proof. (a) If we consider $v_{h}=b \in K_{h}$ in the discrete elliptic variational inequality (10) we have

$$
\begin{aligned}
\lambda\left\|u_{h g}-b\right\|_{V}^{2} & \leq a\left(u_{h g}-b, u_{h g}-b\right) \\
& \leq\left(g, u_{h g}-b\right)_{H}+\left(q, b-u_{h g}\right)_{Q} \\
& \leq\left(\|g\|_{H}+\|q\|_{Q}\left\|\gamma_{0}\right\|\right)\left\|u_{h g}-b\right\|_{V},
\end{aligned}
$$

where $\gamma_{0}$ is the trace operator and therefore (11) holds.

(b) As $u_{h g_{1}}$ and $u_{h g_{2}}$ are, respectively, the solutions of discrete elliptic variational inequalities (10) for $g_{1}$ and $g_{2}$, we have

$$
\begin{aligned}
a\left(u_{h g_{i}}, v_{h}-u_{h g_{i}}\right) \geq & \left(g_{i}, v_{h}-u_{h g_{i}}\right)_{H} \\
& -\left(q, v_{h}-u_{h g_{i}}\right)_{Q}, \quad \forall v_{h} \in K_{h}
\end{aligned}
$$


for $i=1,2$. By coerciveness of $a$ we deduce

$$
\begin{aligned}
\lambda\left\|u_{h g_{2}}-u_{h g_{1}}\right\|_{V}^{2} & \leq a\left(u_{h g_{2}}-u_{h g_{1}}, u_{h g_{2}}-u_{h g_{1}}\right) \\
& \leq\left(g_{2}-g_{1}, u_{h g_{2}}-u_{h g_{1}}\right)_{H} \\
& \leq\left\|g_{2}-g_{1}\right\|_{H}\left\|u_{h g_{2}}-u_{h g_{1}}\right\|_{V}
\end{aligned}
$$

$\forall h>0$;

thus (12) holds.

(c) Let $h>0$. From item (a) we have that $\left\|u_{h g_{n}}\right\| \leq C \forall n$; then there exist $\eta \in V$ such that $u_{h g_{n}} \rightarrow \eta$ in $V$ weak (in $H$ strong). If we consider the discrete elliptic inequality (10) we have

$$
\begin{aligned}
a\left(u_{h g_{n}}, v_{h}-u_{h g_{n}}\right) \geq & \left(g_{n}, v_{h}-u_{h g_{n}}\right)_{H} \\
& -\left(q, v_{h}-u_{h g_{n}}\right)_{Q}
\end{aligned}
$$

and using the fact that $a$ is a lower weak semicontinuous application then, when $n$ goes to infinity, we obtain that

$$
a\left(\eta, v_{h}-\eta\right) \geq\left(g, v_{h}-\eta\right)_{H}-\left(q, v_{h}-\eta\right)_{Q}
$$

and from uniqueness of the solution of problem $\left(S_{h}\right)$, we deduce that $\eta=u_{h g} \in K_{h}$.

Now, it is easily to see that

$$
a\left(u_{h g_{n}}-u_{h g}, u_{h g_{n}}-u_{h g}\right) \leq-\left(g-g_{n}, u_{h g_{n}}-u_{h g}\right)_{H}
$$

and from the coerciveness of $a$ we obtain

$$
\lambda\left\|u_{h g_{n}}-u_{h g}\right\|_{V}^{2} \leq\left(g-g_{n}, u_{h g_{n}}-u_{h g}\right)_{H} .
$$

As $u_{h g_{n}} \rightarrow u_{h g}$ in $H$ and $g_{n} \rightarrow g$ in $H$, by passing to the limit when $n \rightarrow \infty$ in the previous inequality, we obtain

$$
\lim _{n \rightarrow \infty}\left\|u_{h g_{n}}-u_{g}\right\|_{V}=0
$$

Henceforth we will consider the following definitions [2]: given $\mu \in[0,1]$ and $g_{1}, g_{2} \in H$, we have the convex combinations of two data items

$$
g_{3}(\mu)=\mu g_{1}+(1-\mu) g_{2} \in H,
$$

the convex combination of two discrete solutions

$$
u_{h 3}(\mu)=\mu u_{h g_{1}}+(1-\mu) u_{h g_{2}} \in K_{h},
$$

and we define $u_{h 4}(\mu)$ as the associated state system which is the solution of the discrete elliptic variational inequality (10) for the control $g_{3}(\mu)$.

Then, we have the following properties.

Lemma 3. Given the controls $g_{1}, g_{2} \in H$, one has that

(a)

$$
\begin{aligned}
\left\|u_{h 3}\right\|_{H}^{2}= & \mu\left\|u_{h g_{1}}\right\|_{H}^{2}+(1-\mu)\left\|u_{h g_{2}}\right\|_{H}^{2} \\
& -\mu(1-\mu)\left\|u_{h g_{2}}-u_{h g_{1}}\right\|_{H}^{2},
\end{aligned}
$$

(b)

$$
\begin{aligned}
\left\|g_{3}(\mu)\right\|_{H}^{2}= & \mu\left\|g_{1}\right\|_{H}^{2}+(1-\mu)\left\|g_{2}\right\|_{H}^{2} \\
& -\mu(1-\mu)\left\|g_{2}-g_{1}\right\|_{H}^{2} .
\end{aligned}
$$

Proof. (a) From definition (22) we get

$$
\begin{aligned}
\left\|u_{h 3}\right\|_{H}^{2}= & \mu^{2}\left\|u_{h g_{1}}\right\|_{H}^{2}+(1-\mu)^{2}\left\|u_{h g_{2}}\right\|_{H}^{2} \\
& +2 \mu(1-\mu)\left(u_{h g_{1}}, u_{h g_{2}}\right)_{H}, \\
\left\|u_{h g_{2}}-u_{h g_{1}}\right\|_{H}^{2}= & \left\|u_{h g_{2}}\right\|_{H}^{2}+\left\|u_{h g_{1}}\right\|_{H}^{2} \\
& -2\left(u_{h g_{1}}, u_{h g_{2}}\right)_{H},
\end{aligned}
$$

and then we conclude (23).

(b) It follows from a similar method to part (a).

Theorem 4. If $u_{g}$ and $u_{h g}$ are the solutions of the elliptic variational inequalities (3) and (10), respectively, for the control $g \in H$, then $u_{h g} \rightarrow u_{g}$ in $V$ strong when $h \rightarrow 0^{+}$.

Proof. From Lemma 2 we have that there exists a constant $C>0$ independent of $h$ such that $\left\|u_{h g}\right\|_{V} \leq C \forall h>0$, and then we conclude that there exists $\eta \in V$ so that $u_{h g} \rightarrow \eta$ in $V$ weak as $h \rightarrow 0^{+}$and $\eta \in K$. On the other hand, given $v \in K$ there exist $v_{h}^{*}$ such that $v_{h}^{*} \in K_{h}$ for each $h$ and $v_{h}^{*} \rightarrow v$ in $V$ strong when $h$ goes to zero. Now, by considering $v_{h}^{*} \in K_{h}$ in the discrete elliptic variational inequality (10) we get

$$
\begin{aligned}
a\left(u_{h g}, u_{h g}\right) \leq & a\left(u_{h g}, v_{h}^{*}\right)-\left(g, v_{h}^{*}-u_{h g}\right) \\
& +\left(q, v_{h}^{*}-u_{h g}\right)_{\mathrm{Q}}
\end{aligned}
$$

and when we pass to the limit as $h \rightarrow 0^{+}$in (26) by using the fact that bilinear form $a$ is lower weak semicontinuous in $V$ we obtain

$$
a(\eta, \eta) \leq a(\eta, v)-(g, v-\eta)+(q, v-\eta)_{Q} ;
$$

that it is to say,

$$
a(\eta, v-\eta) \geq(g, v-\eta)-(q, v-\eta)_{\mathrm{Q}} \quad \forall v \in K
$$

and, from the uniqueness of the solution of the discrete elliptic variational inequality (3), we obtain that $\eta=u_{g}$.

Now, we will prove the strong convergence. If we consider $v=u_{h g} \in K_{h} \subset K$ in the elliptic variational inequality (3) and $v_{h}=\Pi_{h}\left(u_{g}\right) \in K_{h}$ in (10), from the coerciveness of $a$ and by some mathematical computation, we obtain that

$$
\begin{aligned}
\lambda\left\|u_{h g}-u_{g}\right\|_{V}^{2} \leq & a\left(u_{h g}-u_{g}, u_{h g}-u_{g}\right) \\
\leq & a\left(u_{h g}, \Pi_{h}\left(u_{g}\right)-u_{g}\right) \\
& -\left(g, \Pi_{h}\left(u_{g}\right)-u_{g}\right) \\
& +\left(q, \Pi_{h}\left(u_{g}\right)-u_{g}\right)_{Q} ;
\end{aligned}
$$

then by passing to the limit when $h \rightarrow 0^{+}$it results in $\lim _{h \rightarrow 0^{+}}\left\|u_{h g}-u_{g}\right\|_{V}=0$. 


\section{Discretization of the Optimal Control Problem}

Now, we consider the continuous optimal control problem which was established in (6). The associated discrete cost functional $J_{h}: H \rightarrow \mathbb{R}_{0}^{+}$is defined by the following expression:

$$
J_{h}(g)=\frac{1}{2}\left\|u_{h g}\right\|_{H}^{2}+\frac{M}{2}\|g\|_{H}^{2}
$$

and we establish the discrete optimal control problem $\left(P_{h}\right)$ as follows: find $g_{\mathrm{op}_{h}} \in H$ such that

$$
J_{h}\left(g_{\mathrm{op}_{h}}\right)=\min _{g \in H} J_{h}(g)
$$

where $u_{h g}$ is the associated state system solution of the problem $\left(S_{h}\right)$ which was described for the discrete elliptic variational inequality (10) for a given control $g \in H$.

Theorem 5. Given the control $g \in H$, one has

(a)

$$
\lim _{\|g\|_{H} \rightarrow \infty} J_{h}(g)=\infty
$$

(b) $J_{h}(g) \geq(M / 2)\|g\|_{H}^{2}-C\|g\|_{H}$ for some constant $C$ independent of $h$;

(c) the functional $J_{h}$ is a lower weakly semicontinuous application in $\mathrm{H}$;

(d) there exists a solution of the discrete optimal control problem (31) for all $h>0$.

Proof. (a) From the definition of $J_{h}(g)$ we obtain (a) and (b).

(c) Let $g_{n} \rightarrow g$ in $H$ weak; then by using the equality $\left\|g_{n}\right\|_{H}^{2}=\left\|g_{n}-g\right\|_{H}^{2}-\|g\|_{H}^{2}+2\left(g_{n}, g\right)_{H}$ we obtain that $\|g\|_{H} \leq$ $\liminf _{n \rightarrow \infty}\left\|g_{n}\right\|_{H}^{2}$. Therefore, we have

$$
\liminf _{n \rightarrow \infty} J_{h}\left(g_{n}\right) \geq \frac{1}{2}\left\|u_{h g}\right\|_{H}^{2}+\frac{M}{2}\|g\|_{H}^{2}=J_{h}(g) .
$$

(d) It follows from [4].

Lemma 6. If the continuous state system has the regularity $u_{g} \in H^{r}(\Omega)(1<r \leq 2)$ then one has the following estimations $\forall g \in H$ :

(a)

$$
\left\|u_{h g}-u_{g}\right\|_{V} \leq C h^{(r-1) / 2},
$$

(b)

$$
\left|J_{h}(g)-J(g)\right| \leq C h^{(r-1) / 2}
$$

where C's are constants independent of $h$.
Proof. (a) As $u_{g} \in K$, we have that $\Pi_{h}\left(u_{g}\right) \in K_{h} \subset K$. If we consider $v_{h}=\Pi_{h}\left(u_{g}\right)$ in (10), by using the inequalities (29), we obtain

$$
\begin{aligned}
\lambda\left\|u_{h g}-u_{g}\right\|_{V}^{2} \leq & a\left(u_{h g}-u_{g}, u_{h g}-u_{g}\right) \\
\leq & a\left(u_{h g}, \Pi_{h}\left(u_{g}\right)-u_{g}\right) \\
& -\left(g, \Pi_{h}\left(u_{g}\right)-u_{g}\right) \\
& +\left(q, \Pi_{h}\left(u_{g}\right)-u_{g}\right)_{\mathrm{Q}} \\
\leq & C\left\|\Pi_{h}\left(u_{g}\right)-u_{g}\right\|_{V} \leq C\left\|u_{g}\right\|_{r} h^{r-1} \\
\leq & C h^{r-1},
\end{aligned}
$$

and then (34) holds.

(b) From the definitions of $J$ and $J_{h}$, it results in

$$
\begin{aligned}
J_{h}(g)-J(g) & =\frac{1}{2}\left(\left\|u_{h g}\right\|_{H}^{2}-\left\|u_{g}\right\|_{H}^{2}\right) \\
& =\frac{1}{2}\left[\left\|u_{h g}-u_{g}\right\|_{H}^{2}+\left(u_{g}, u_{h g}-u_{g}\right)\right]
\end{aligned}
$$

and therefore

$$
\begin{aligned}
& \left|J_{h}(g)-J(g)\right| \\
& \quad \leq\left(\frac{1}{2}\left\|u_{h g}-u_{g}\right\|_{H}+\left\|u_{g}\right\|_{H}\right)\left\|u_{h g}-u_{g}\right\|_{H} \\
& \quad \leq C h^{(r-1) / 2} .
\end{aligned}
$$

Following the idea given in [2] we define an open problem: given the controls $g_{1}, g_{2} \in H$ and $\forall \mu \in[0,1]$, $\forall h>0$

$$
\begin{aligned}
0 & \leq u_{h 4}(\mu) \leq u_{h 3}(\mu) \quad \text { in } \Omega, \\
\left\|u_{h 4}(\mu)\right\|_{H} & \leq\left\|u_{h 3}(\mu)\right\|_{H} .
\end{aligned}
$$

Remark 7. We have that $(39) \Rightarrow(40)$.

Remark 8. The equivalent inequality (39) for the continuous optimal control problem $(P)$ is true; that is [2], for all $g_{1}, g_{2} \in$ $H$, and $\forall \mu \in[0,1]$,

$$
0 \leq u_{4}(\mu) \leq u_{3}(\mu) \quad \text { in } \Omega \text {, }
$$

where $u_{3}(\mu)=\mu u_{g_{1}}+(1-\mu) u_{g_{2}} \in K, u_{g_{i}}(i=1,2)$ is the unique solution of the elliptic variational inequality (3) when we consider $g_{i}$ instead of $g$ and $u_{4}(\mu)$ is the unique solution of the elliptic variational inequality $(3)$ when we consider $g_{3}(\mu)$ instead of $g$. 
Remark 9. If (40) (or (39)) is true, then the functional $J_{h}$ is $H$-elliptic and a strictly convex application because we have

$$
\begin{aligned}
\mu J_{h}\left(g_{1}\right)+(1-\mu) J_{h}\left(g_{2}\right)-J_{h}\left(g_{3}(\mu)\right) \\
=\frac{\mu(1-\mu)}{2}\left[\left\|u_{h g_{2}}-u_{h g_{1}}\right\|_{H}^{2}+M\left\|g_{2}-g_{1}\right\|_{H}^{2}\right] \\
\quad+\frac{1}{2}\left[\left\|u_{h 3}\right\|_{H}^{2}-\left\|u_{h 4}\right\|_{H}^{2}\right] \\
\geq \frac{\mu(1-\mu)}{2}\left[\left\|u_{h g_{2}}-u_{h g_{1}}\right\|_{H}^{2}+M\left\|g_{2}-g_{1}\right\|_{H}^{2}\right]>0
\end{aligned}
$$

and therefore, the uniqueness for the discrete optimal control problem $\left(P_{h}\right)$ holds in Theorem 5 .

Now, we will show the convergence result for optimal control problems governed by elliptic variational inequalities in order to generalize the result for optimal control problems governed by elliptic variational equalities [19]. We remark that there exist a few numbers of papers for the numerical analysis of optimal control problems governed by elliptic variational inequalities, for example [20-22].

Theorem 10. Let $u_{g_{o p}} \in K$ be the continuous state system associated with the optimal control $g_{o p} \in H$ which is the solution of the continuous distributed optimal control problem (6). If, for each $h>0$, one chooses an optimal control $g_{o p_{h}} \in H$ which is the solution of the discrete distributed optimal control problem (31) and its corresponding discrete state system $u_{h g_{o p_{h}}} \in$ $K_{h}$, one obtains that

$$
\begin{aligned}
u_{h g_{o p_{h}}} & \longrightarrow u_{g_{o p}} \text { on } V \text { strong, } \\
g_{o p_{h}} & \longrightarrow g_{o p} \quad \text { on } H \text { strong when } h \longrightarrow 0^{+} .
\end{aligned}
$$

Proof. Let $h>0$ and let $g_{\mathrm{op}_{h}}$ be a solution of (31), and let $u_{h g_{\text {oph }}}$ be its associated discrete optimal state system which is the solution of the discrete elliptic variational inequality (10) for each $h>0$. From (30) we have that for all $g \in H$

$$
\begin{aligned}
J_{h}\left(g_{\mathrm{op}_{h}}\right) & =\frac{1}{2}\left\|u_{h g_{\mathrm{op}_{h}}}\right\|_{H}^{2}+\frac{M}{2}\left\|g_{\mathrm{op}_{h}}\right\|_{H}^{2} \\
& \leq \frac{1}{2}\left\|u_{h g}\right\|_{H}^{2}+\frac{M}{2}\|g\|_{H}^{2} .
\end{aligned}
$$

Then, if we consider $g=0$ and $u_{h 0}$ its corresponding associated state system, it results in the following:

$$
J_{h}\left(g_{\mathrm{op}_{h}}\right)=\frac{1}{2}\left\|u_{h g_{\mathrm{op}_{h}}}\right\|_{H}^{2}+\frac{M}{2}\left\|g_{\mathrm{op}_{h}}\right\|_{H}^{2} \leq \frac{1}{2}\left\|u_{h 0}\right\|_{H}^{2} .
$$

From Lemma 2 we have that $\left\|u_{h 0}\right\|_{H} \leq C \forall h$; then we can obtain

$$
\begin{aligned}
\left\|u_{h g_{\mathrm{op}_{h}}}\right\|_{H} & \leq C \quad \forall h>0, \\
\left\|g_{\mathrm{op}_{h}}\right\|_{H} & \leq \frac{1}{\sqrt{M}}\left\|u_{h 0}\right\|_{H} \leq \frac{C}{\sqrt{M}} \quad \forall h>0 .
\end{aligned}
$$

If we consider $v_{h}=b \in K_{h}$ in inequality (10) for $g_{\mathrm{op}_{h}}$, we obtain

$$
\begin{aligned}
a\left(u_{h g_{\mathrm{op}_{h}}}, b-u_{h g_{\mathrm{op}_{h}}}\right) \geq & \left(g_{\mathrm{op}_{h}}, b-u_{h g_{\mathrm{op}_{h}}}\right) \\
& -\left(q, b-u_{h g_{\mathrm{op}_{h}}}\right)_{\mathrm{Q}} ;
\end{aligned}
$$

therefore

$$
\begin{aligned}
& a\left(u_{h g_{\mathrm{op}_{h}}}-b, u_{h_{\mathrm{op}_{h}}}-b\right) \\
& \quad \leq\left(g_{\mathrm{op}_{h}}, u_{h g_{\mathrm{op}_{h}}}-b\right)-\left(q, u_{h g_{\mathrm{op}_{h}}}-b\right)_{\mathrm{Q}},
\end{aligned}
$$

and from the coerciveness of the application $a$ we have that $\left\|u_{h g_{\text {op }}}-b\right\|_{V} \leq C$ and in consequence $\left\|u_{h g_{\text {op }_{h}}}\right\|_{V} \leq C$.

Now we can say that there exist $\eta \in V$ and $f \in H$ such that $u_{h g_{\mathrm{op}_{h}}} \rightarrow \eta$ in $V$ weak (in $H$ strong), and $g_{\mathrm{op}_{h}} \rightarrow f$ in $H$ weak when $h \rightarrow 0^{+}$. Then, $\eta / \Gamma_{1}=b$ and $\eta \geq 0$ in $\Omega$; that is, $\eta \in K$.

Letting $v \in K$, there exist $v_{h} \in K_{h}$ such that $v_{h} \rightarrow v$ in $V$ strong when $h \rightarrow 0^{+}$. Then, if we consider the variational elliptic inequality (10) for $g=g_{\mathrm{op}_{h}}$ we have

$$
\begin{aligned}
a\left(u_{h g_{\mathrm{op}_{h}}}, v_{h}\right) \geq & a\left(u_{h g_{\mathrm{op}_{h}}}, u_{h g_{\mathrm{op}_{h}}}\right)+\left(g_{\mathrm{op}_{h}}, v_{h}-u_{h g_{\mathrm{op}_{h}}}\right) \\
& -\left(q, v_{h}-u_{h g_{\mathrm{op}_{h}}}\right)_{\mathrm{Q}} .
\end{aligned}
$$

Taking into account that the application $a$ is a lower weak semicontinuous application in $V$ and by passing to the limit when $h$ goes to zero in (49) we obtain that

$$
a(\eta, v-\eta) \geq(f, v-\eta)-(q, v-\eta)_{\mathrm{Q}}, \quad \forall v \in K
$$

and by the uniqueness of the solution of the problem given by the elliptic variational inequality (3), we deduce that $\eta=u_{f}$.

Finally, the norm on $H$ is a lower semicontinuous application in the weak topology; then we can prove that

$$
\begin{aligned}
J(f) & =\frac{1}{2}\left\|u_{f}\right\|_{H}^{2}+\frac{M}{2}\|f\|_{H}^{2} \leq \liminf _{h \rightarrow 0^{+}} J_{h}\left(g_{\mathrm{op}_{h}}\right) \\
& \leq \liminf _{h \rightarrow 0^{+}} J_{h}(g)=\frac{1}{2} \lim _{h \rightarrow 0^{+}}\left\|u_{h g}\right\|_{H}^{2}+\frac{M}{2}\|g\|_{H}^{2} \\
& =\frac{1}{2}\left\|u_{g}\right\|_{H}^{2}+\frac{M}{2}\|g\|_{H}^{2}=J(g), \quad \forall g \in H
\end{aligned}
$$

and because of the uniqueness of the optimal problem (6), it results in $f=g_{\mathrm{op}}$ and $\eta=u_{g_{\mathrm{op}}}$.

Now, if we consider $v=u_{h g_{\mathrm{op}_{h}}} \in K_{h} \subset K$ in the elliptic variational inequality (3) for the control $g_{\text {op }}$ and we define $z_{h}=u_{h g_{\mathrm{op}}}-u_{g_{\mathrm{op}}}$, we have that

$$
\begin{aligned}
a\left(z_{h}, z_{h}\right) \leq & a\left(u_{h g_{\mathrm{op} h}}, u_{h g_{\mathrm{op} h}}\right)-a\left(u_{h g_{\mathrm{op} h}}, u_{g_{\mathrm{op}}}\right) \\
& -\left(g_{\mathrm{op}}, u_{h g_{\mathrm{op} h}}-u_{g_{\mathrm{op}}}\right) \\
& +\left(q, u_{h g_{\mathrm{op} h}}-u_{g_{\mathrm{op}}}\right)_{\mathrm{Q}},
\end{aligned}
$$


and by considering $v=\Pi_{h}\left(u_{g_{\mathrm{op}}}\right) \in K_{h}$ for $g=g_{\mathrm{op}_{h}}$ in inequality (10) we obtain

$$
\begin{aligned}
a\left(u_{h g_{\mathrm{op}_{h}}}, u_{h g_{\mathrm{op}_{h}}}\right) \leq & -\left(g_{\mathrm{op}_{h}}, \Pi_{h}\left(u_{g_{\mathrm{op}}}\right)-u_{h g_{\mathrm{op}_{h}}}\right) \\
& +\left(q, \Pi_{h}\left(u_{g_{\mathrm{op}}}\right)-u_{h g_{\mathrm{op}_{h}}}\right)_{\mathrm{Q}} \\
& +a\left(u_{h g_{\mathrm{op}_{h}}}, \Pi_{h}\left(u_{\mathrm{gop}_{\mathrm{op}}}\right)\right)
\end{aligned}
$$

and then by the coerciveness of $a$ we get

$$
\begin{aligned}
\lambda\left\|z_{h}\right\|_{V}^{2} \leq & \left(q, \Pi_{h}\left(u_{g_{\mathrm{op}}}\right)-u_{g_{\mathrm{op}}}\right)_{\mathrm{Q}} \\
& +a\left(u_{h g_{\mathrm{op} h}}, \Pi_{h}\left(u_{g_{\mathrm{op}}}\right)-u_{g_{\mathrm{op}}}\right) \\
& +\left(g_{\mathrm{op}_{h}}-g_{\mathrm{op}}, u_{h g_{\mathrm{op}}}-u_{g_{\mathrm{op}}}\right) \\
& -\left(g_{\mathrm{op}}, \Pi_{h}\left(u_{g_{\mathrm{op}}}\right)-u_{g_{\mathrm{op}}}\right) .
\end{aligned}
$$

When we pass to the limit as $h \rightarrow 0$ in (54) and by using the strong convergence of $u_{h g_{\text {op }}}$ to $u_{g_{\text {op }}}$ on $H$ and the weak convergence of $g_{\mathrm{op}_{h}}$ to $g_{\mathrm{op}}$ on $H$, we have

$$
\lim _{h \rightarrow 0^{+}}\left\|u_{g_{\mathrm{op}}}-u_{h g_{\mathrm{op} h}}\right\|_{V}=0
$$

The strong convergence of the optimal controls $g_{\mathrm{op}}$ to $g_{\mathrm{op}}$ is obtained by using Theorem 5 and $g_{\mathrm{op}_{h}} \rightarrow g_{\mathrm{op}}$ weakly on $H$; that is,

$$
\begin{aligned}
J\left(g_{\mathrm{op}}\right) & =\frac{1}{2}\left\|u_{g_{\mathrm{op}}}\right\|_{H}^{2}+\frac{M}{2}\left\|g_{\mathrm{op}}\right\|_{H}^{2} \leq \liminf _{h \rightarrow 0^{+}} J_{h}\left(g_{\mathrm{op}_{h}}\right) \\
& \leq \liminf _{h \rightarrow 0^{+}} J_{h}\left(g_{\mathrm{op}}\right) \\
& =\liminf _{h \rightarrow 0^{+}} \frac{1}{2}\left\|u_{g_{\mathrm{op}}}\right\|_{H}^{2}+\frac{M}{2}\left\|g_{\mathrm{op}}\right\|_{H}^{2}=J\left(g_{\mathrm{op}}\right) ;
\end{aligned}
$$

then $\lim _{h \rightarrow 0}\left\|g_{\mathrm{op}_{h}}\right\|_{H}=\left\|g_{\mathrm{op}}\right\|_{H}$ and therefore $\lim _{h \rightarrow 0^{+}}\left\|g_{\mathrm{op}_{h}}-g_{\mathrm{op}}\right\|_{H}=0$.

\section{Conclusions}

We have proved the convergence of a discrete optimal control and its corresponding discrete state system governed by a discrete elliptic variational inequality to the continuous optimal control and its corresponding continuous state system which is also governed by a continuous elliptic variational inequality by using the finite element method with Lagrange's triangles of type 1 .

\section{Conflict of Interests}

The authors declare that there is no conflict of interests regarding the publication of this paper.

\section{Acknowledgments}

This paper has been partially sponsored by Project PIP no. 0534 from CONICET-UA, Rosario, Argentina, and AFOSRSOARD Grant FA9550-14-1-0122.

\section{References}

[1] D. Kinderhlerer and G. Stampacchia, An Introduction to Variational Inequalities and Their Applications, Academic Press, New York, NY, USA, 1980.

[2] M. Boukrouche and D. A. Tarzia, "Convergence of distributed optimal control problems governed by elliptic variational inequalities," Computational Optimization and Applications, vol. 53, no. 2, pp. 375-393, 2012.

[3] V. Barbu, Optimal Control of Variational Inequalities, vol. 100 of Research Notes in Mathematics, Pitman, London, UK, 1984.

[4] J.-L. Lions, Contrôle Optimal de Systémes Gouvernés Par des Équations aux Dérivées Partielles, Dunod, Paris, France, 1968.

[5] F. Tröltzsch, Optimal Control of Partial Differential Equations, American Mathematical Society, Providence, RI, USA, 2010.

[6] K. Ait Hadi, "Optimal control of the obstacle problem: optimality conditions," IMA Journal of Mathematical Control and Information, vol. 23, no. 3, pp. 325-334, 2006.

[7] M. Bergounioux, "Optimal control of an obstacle problem," Applied Mathematics and Optimization, vol. 36, no. 2, pp. 147$172,1997$.

[8] J. C. De los Reyes and C. Meyer, "Strong stationarity conditions for a class of optimization problems governed by variational inequalities of the second kind," Journal of Optimization Theory and Applications, pp. 1-35, 2015.

[9] C. M. Gariboldi and D. A. Tarzia, "Convergence of distributed optimal controls on the internal energy in mixed elliptic problems when the heat transfer coefficient goes to infinity," Applied Mathematics and Optimization, vol. 47, no. 3, pp. 213230, 2003.

[10] K. Ito and K. Kunisch, "Optimal control of elliptic variational inequalities," Applied Mathematics and Optimization, vol. 41, no. 3, pp. 343-364, 2000.

[11] F. Mignot, "Control dans les inequations variationelles elliptiques," Journal of Functional Analysis, vol. 22, pp. 130-185, 1976.

[12] F. Mignot and J.-P. Puel, "Optimal control in some variational inequalities," SIAM Journal on Control and Optimization, vol. 22, no. 3, pp. 466-476, 1984.

[13] E. Casas and M. Mateos, "Uniform convergence of the FEM. Applications to state constrained control problems," Computational \& Applied Mathematics, vol. 21, no. 1, pp. 67-100, 2002.

[14] R. S. Falk, "Error estimates for the approximation of a class of variational inequalities," Mathematics and Computers in Simulation, vol. 28, pp. 963-971, 1974.

[15] M. Hinze, "Discrete concepts in PDE constrained optimization," in Optimization with PDE Constraints, M. Hinze, R. Pinnau, R. Ulbrich, and S. Ulbrich, Eds., vol. 23 of Mathematical Modelling: Theory and Applications, chapter 3, pp. 157-232, Springer, Dordrecht, The Netherlands, 2009.

[16] D. A. Tarzia, "Numerical analysis for the heat flux in a mixed elliptic problem to obtain a discrete steady-state two-phase Stefan problem," SIAM Journal on Numerical Analysis, vol. 33, no. 4, pp. 1257-1265, 1996.

[17] S. Brenner and L. Scott, The Mathematical Theory of Finite Elements, Springer, Berlin, Germany, 1994. 
[18] P. Ciarlet, The Finite Element Method for Elliptic Problems, SIAM, Philadelphia, Pa, USA, 2002.

[19] D. A. Tarzia, "A commutative diagram among discrete and continuous Neumann boundary optimal control problems," Advances in Differential Equations and Control Processes, vol. 14, no. 1, pp. 23-54, 2014.

[20] J. Haslinger and T. Roubicek, "Optimal control of variational inequalities. Approximation theory and numerical realization," Applied Mathematics and Optimization, vol. 14, no. 3, pp. 187201, 1986.

[21] M. Hintermüller, "An active-set equality constrained Newton solver with feasibility restoration for inverse coefficient problems in elliptic variational inequalities," Inverse Problems, vol. 24, no. 3, Article ID 034017, 23 pages, 2008.

[22] C. Meyer and O. Thoma, "A priori finite element error analysis for optimal control of the obstacle problem," SIAM Journal on Numerical Analysis, vol. 51, no. 1, pp. 605-628, 2013. 


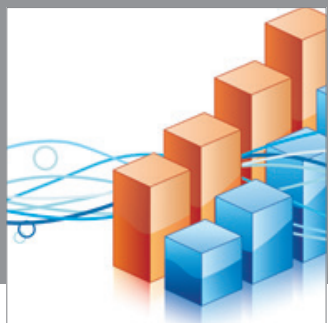

Advances in

Operations Research

mansans

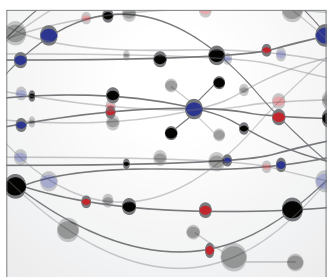

The Scientific World Journal
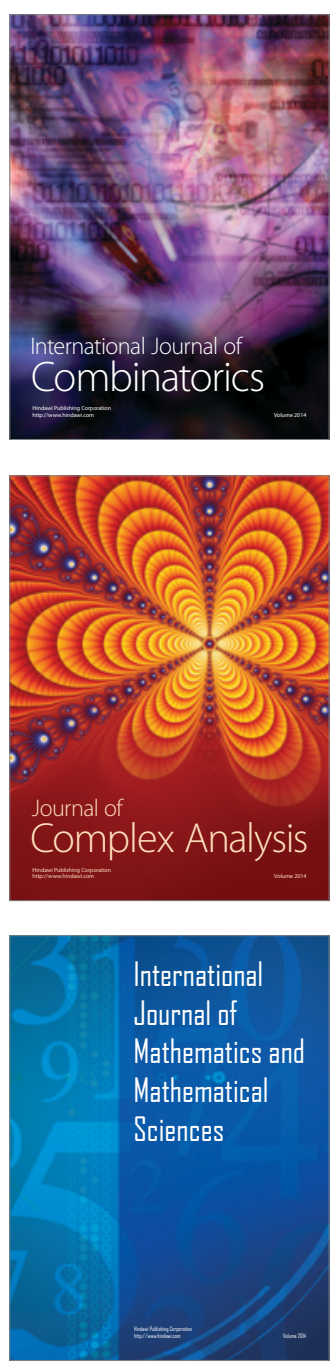
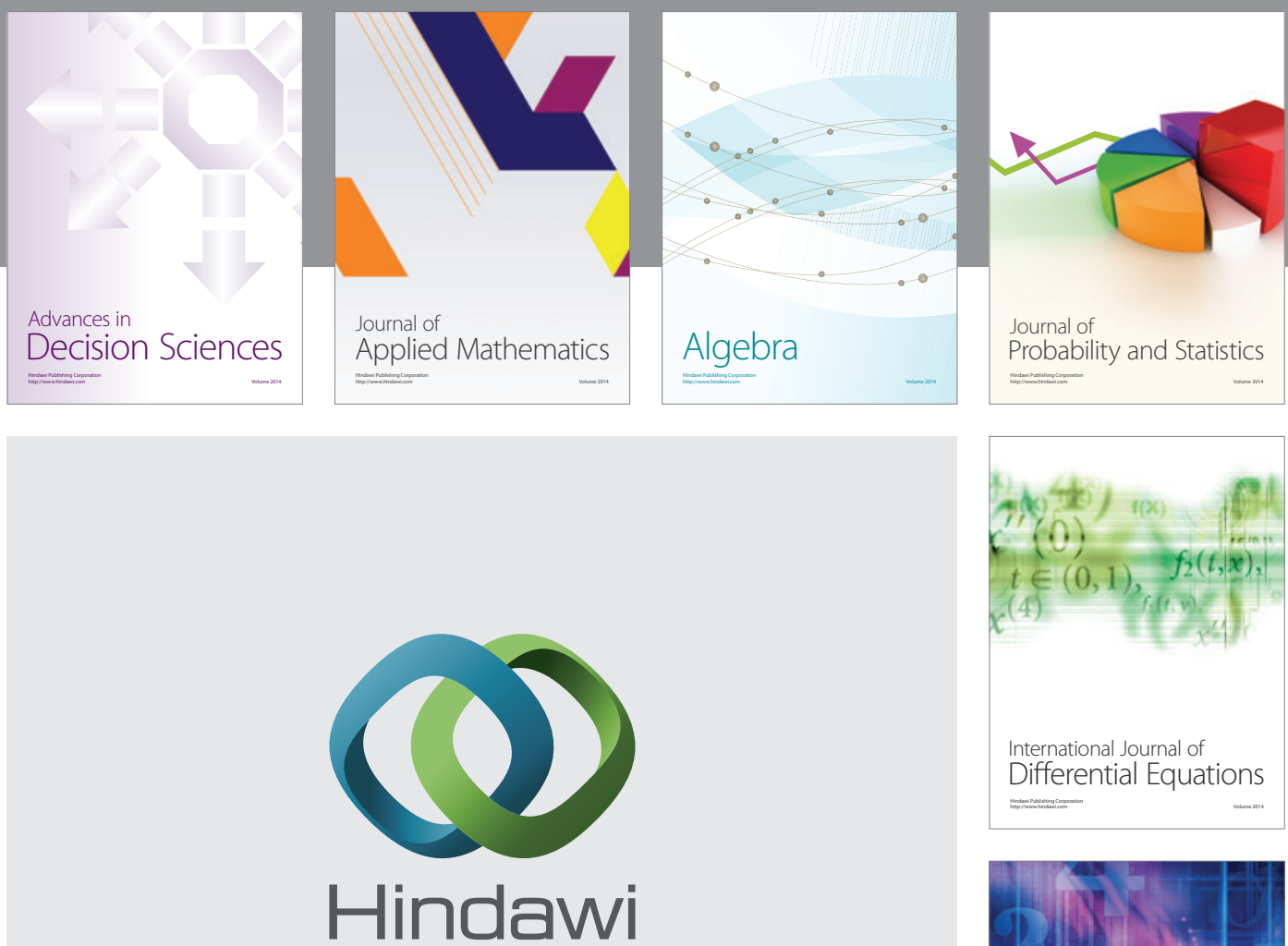

Submit your manuscripts at http://www.hindawi.com
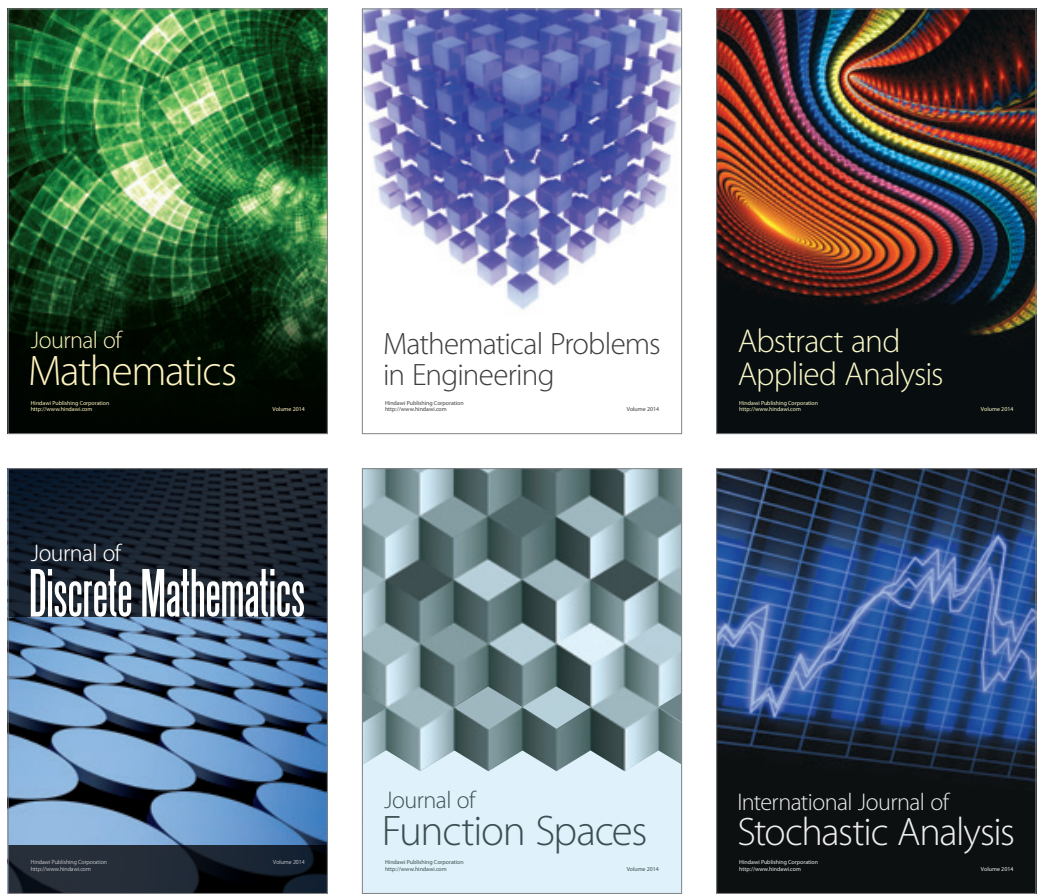

Journal of

Function Spaces

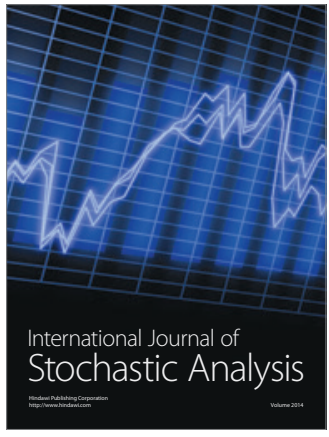

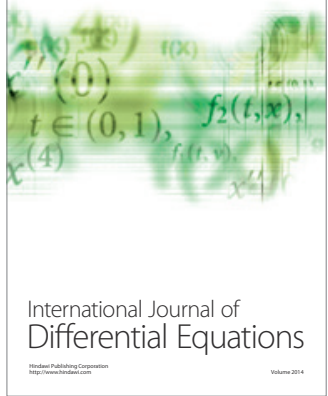
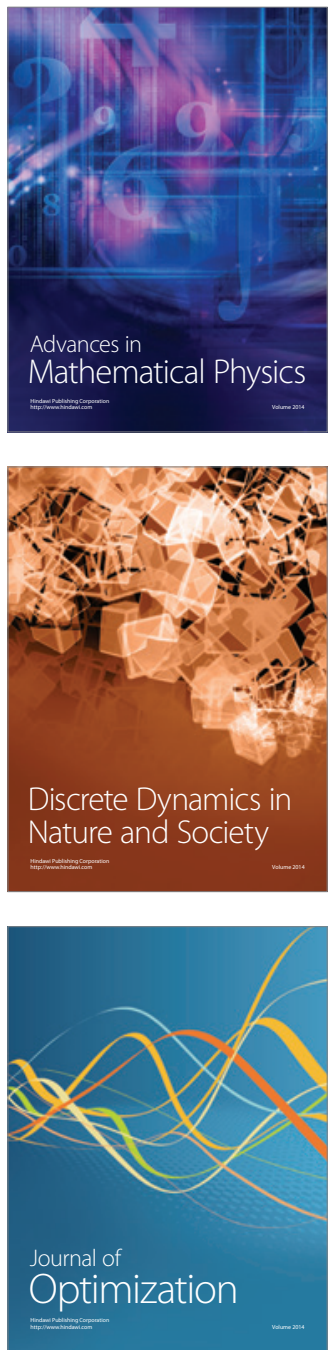